\title{
A CIRCULAÇÃO EXTRACORPÓREA EM CASOS DE DISSECÇÃO AÓRTICA
}

\section{Camila Silva Moreno; Aldenira Matias de Moura1; Murilo Barros Silveira²}

${ }^{1}$ Biomédica; Pós-graduada em Perfusão/Instituto Asgard; Goiânia-GO, Brasil. 2 Biomédico. Especialista em Microbiologia. Mestre e Doutorando em Biologia da Relação Parasito-Hospedeiro/Universidade Federal de Goiás. Goiânia-GO, Brasil. murilo_bsilveira@hotmail.com

Recebido em: 15/05/2021 - Aprovado em: 15/06/2021 - Publicado em: 30/06/2021 DOI: 10.18677/EnciBio_2021B1

Introdução: A dissecção da aorta ocorre quando o dano à camada mais interna da aorta faz com que o sangue passe entre as várias camadas da parede aórtica e force a separação dessas camadas. A redução do suprimento de sangue para outros órgãos pode causar acidente vascular cerebral ou isquemia mesentérica. Sabendo da importância da artéria aorta, da complexidade, seriedade e extensão dos danos que pode ocasionar, torna-se cada vez mais necessário o estudo, pesquisa e desenvolvimento de novas técnicas e alternativas de tratamento. Objetivo: Descrever a função da circulação extracorpórea em casos de dissecção da artéria aorta. Metodologia: Constitui uma revisão bibliográfica descritiva realizada nas bases de dados eletrônicas PubMed e SciELO, através da consulta a artigos publicados entre os anos 2000 a 2021. Resultados: Não existe um consenso sobre a canulação arterial mais indicada para cada caso específico quando se trata de dissecção de aorta. Elementos importantes para estratégias de canulação são o estabelecimento rápido de fluxo sistêmico suficiente, cuidados com a má perfusão existente no pré-operatório e prevenção de nova má perfusão induzida pela CEC. As complicações neurológicas não devem ser ignoradas e a mudança para uma canulação diferente pode ser necessária se houver algum sinal de má perfusão. Conclusão: A técnica de canulação varia de acordo com a localização e a extensão da patologia, especialmente em relação a canulação arterial. Com isso verifica-se a necessidade de maiores estudos sobre a perfusão frente aos casos de dissecção de aorta.

PALAVRAS-CHAVE: Artéria aorta; circulação extracorpórea; dissecção de aorta.

\section{EXTRACORPOREAL CIRCULATION IN CASES OF AORTIC DISSECTION}

\begin{abstract}
Introduction: Aortic dissection occurs when damage to the innermost layer of the aorta causes blood to pass between the various layers of the aortic wall and force the separation of these layers. Reducing the blood supply to other organs can cause stroke or mesenteric ischemia. Knowing the importance of the aortic artery, the complexity, seriousness and extent of the damage it can cause, it is increasingly
\end{abstract}


necessary to study, research and develop new techniques and treatment alternatives. Objective: To describe the role of cardiopulmonary bypass in cases of aortic artery dissection. Methodology: It constitutes a descriptive bibliographic review carried out in the electronic databases PubMed and SciELO, through the consultation of articles published between the years 2000 to 2021. Results: There is no consensus on the most suitable arterial cannulation for each specific case when it comes to aortic dissection. Important elements for cannulation strategies are the rapid establishment of sufficient systemic flow, care for preoperative poor perfusion and prevention of new CPB-induced bad perfusion. Neurological complications should not be ignored and a switch to a different cannulation may be necessary if there is any sign of poor perfusion. Conclusion: The cannulation technique varies according to the location and extent of the pathology, especially in relation to arterial cannulation. Thus, there is a need for further studies on perfusion in cases of aortic dissection.

KEYWORDS: Aorta artery; extracorporeal circulation; aortic dissection.

\section{INTRODUÇÃO}

A aorta é a principal artéria do aparelho circulatório, tem origem no ventrículo esquerdo, em continuação com a válvula aórtica (FOX et al., 2015; PLATZZ et al., 2017). Da aorta originam-se as artérias da circulação do músculo cardíaco, as artérias coronárias e as demais artérias da circulação sistêmica que irrigam o crânio, membros superiores, tórax, abdome, pélvis e os membros inferiores. Os distúrbios da aorta estão inclusos os aneurismas e as dissecções (SOUZA; ELIAS, 2006; SCALEA et al., 2019).

O aneurisma da aorta é definido como a dilatação de um ou mais segmentos da mesma que envolva todas as suas camadas, ou seja, intima, média e adventícia. São dilatações irreversíveis com diâmetro transversal excedendo em pelo menos 1,5 vez o seu diâmetro normal (TAN et al., 2017; GARCíA et al., 2018). Podendo ser abdominais ou torácicos. Sendo os aneurismas de aorta abdominais mais frequentes do que os torácicos. A incidência de aneurismas da aorta torácica atinge de seis a oito indivíduos em cada 100 mil pacientes por ano. O diâmetro do aneurisma é o fator mais importante na determinação desse risco. Dentre os aneurismas, a dissecção aórtica é a afecção mais temida da aorta, sendo duas vezes mais frequente que a ruptura da aorta (BUFFOLO et al., 2018). Dissecção de aorta é definida como uma rotura na íntima que expõe uma camada média doente à pressão de pulso na aorta, proporcionando separação de suas camadas, expansão longitudinal e formação de um falso lúmen repleto de sangue (MELVINSDOTTIR et al., 2018).

Em alguns casos, a dissecção começa com a rotura do vaso vasorum, dentro da camada média, formando o hematoma intramural. Algumas doenças da aorta torácica, principalmente as dissecções agudas, podem comprometer a irrigação arterial do cérebro ou da medula, causando lesões isquêmicas capazes de levar ao coma ou resultar em paralisias irreversíveis com monoplegia, hemiplegia ou paraplegia (SEDRAKYAN et al., 2006; MARCACCIO et al., 2018).

A dissecção da aorta torácica é um diagnóstico diferencial importante com síndromes coronarianas agudas. Os pacientes se apresentam com dor torácica aguda irradiada para o pescoço e para as costas, associada com assimetria da amplitude do pulso periférico e eventualmente, choque. O envolvimento da aorta 
ascendente é associado com insuficiência aguda da valva aórtica com bastante frequência, que pode levar a insuficiência aguda do ventrículo esquerdo (KIM et al., 2018). Sendo que a dissecção da aorta ascendente (DAA) tem um péssimo prognóstico se não corrigida com rapidez cirurgicamente, com a ruptura iminente e uma elevada taxa de mortalidade que chega a $1 \%$ por hora quando não tratada (ALMEIDA et al., 2011).

Sabendo da importância da artéria aorta, da complexidade, seriedade e extensão dos danos que a dissecção de aorta pode ocasionar, torna-se cada vez mais necessário o estudo, pesquisa e desenvolvimento de novas técnicas e alternativas de tratamento. Assim, o objetivo do presente estudo foi descrever a função da circulação extracorpórea em casos de dissecção da artéria aorta.

\section{MATERIAIS E MÉTODOS}

Este trabalho constitui uma revisão bibliográfica descritiva realizada nas bases de dados eletrônicas US National Library of Medicine (PubMed) e Scientific Electronic Library Online (SciELO), através da consulta a artigos publicados no período do ano de 2000 ao ano de 2021. Na busca, foram utilizados os seguintes termos contemplados nos Descritores em Ciências da Saúde (DeCS) em língua portuguesa ou inglesa: "aortic dissection" e "cardiopulmonary bypass" de forma isolada ou combinada.

O levantamento bibliográfico foi realizado no período de janeiro a fevereiro do ano de 2021. Foram encontrados inicialmente 48 artigos que demostravam relação com o tema, deste total 13 artigos foram selecionados por apresentarem conteúdos que realmente contribuíram para o cumprimento dos objetivos deste, publicados no período de 2005 a 2020 reforçando o embasamento teórico-conceitual da abordagem em questão. Foram excluídos 35 artigos, pois não apresentavam relação com os objetivos deste estudo, aqueles publicados fora do período temporal adotado e que não apresentavam relação com os objetivos deste estudo.

\section{DISSECÇÃO DE AORTA}

Aneurismas e dissecções constituem as principais doenças da aorta, os quais podem ser submetidos a princípios e técnicas de tratamento cirúrgico comuns. A dissecção aórtica consiste na clivagem ou na separação da camada média da aorta produzida pela penetração do sangue na parede do vaso (COSTA et al., 2019). Forma-se um hematoma progressivo que divide a camada média em duas lâminas que delimitam um falso trajeto para o sangue, denominado falso lúmen repleto de sangue, cuja parede externa é constituída por parte da camada média e pela adventícia da aorta (ISSA et al., 2013; COSTA et al., 2019).

O mecanismo mais comum consiste na laceração espontânea da camada íntima e parte da camada média, através da qual o sangue penetra e desgarra as suas camadas. Menos frequentemente, pode ocorrer a rotura dos vasa-vasorum da parede da aorta, que origina um pequeno hematoma intramural que se rompe na luz aórtica e permite a penetração do sangue (RAIVIO et al., 2005; KIM et al., 2018).

A dissecção aórtica pode se limitar a um segmento anatômico, como a aorta ascendente ou a descendente, por exemplo ou pode comprometer toda a extensão do vaso, até a sua bifurcação no abdome. A laceração nas camadas íntima e média da aorta é quase sempre transversal e se localiza 2 a $3 \mathrm{~cm}$ acima do plano da válvula aórtica em cerca de $60 \%$ dos casos (MCCLURE et al., 2018). Em 
aproximadamente $30 \%$ dos casos a laceração da íntima, que inicia a dissecção, ocorre próximo da origem da artéria subclávia esquerda e o plano de clivagem se estende pela aorta descendente, podendo alcançar a bifurcação aórtica e seus ramos (DIAS et al., 2007).

A dissecção que se inicia na aorta descendente também pode progredir no sentido retrógrado e comprometer o arco aórtico e parte da aorta ascendente (Figura 1). Quando a dissecção alcança a área de implantação dos folhetos da válvula aórtica, pode ocorrer o prolapso dos folhetos, que resulta em insuficiência aórtica aguda. A progressão do sangue ou do hematoma no interior do falso lúmen pode comprimir ou ocluir a origem dos principais ramos arteriais da aorta e produzir isquemia dos órgãos (FONTES et al., 2005; ISSA et al., 2013).

FIGURA 1. Principais segmentos anatômicos da aorta.

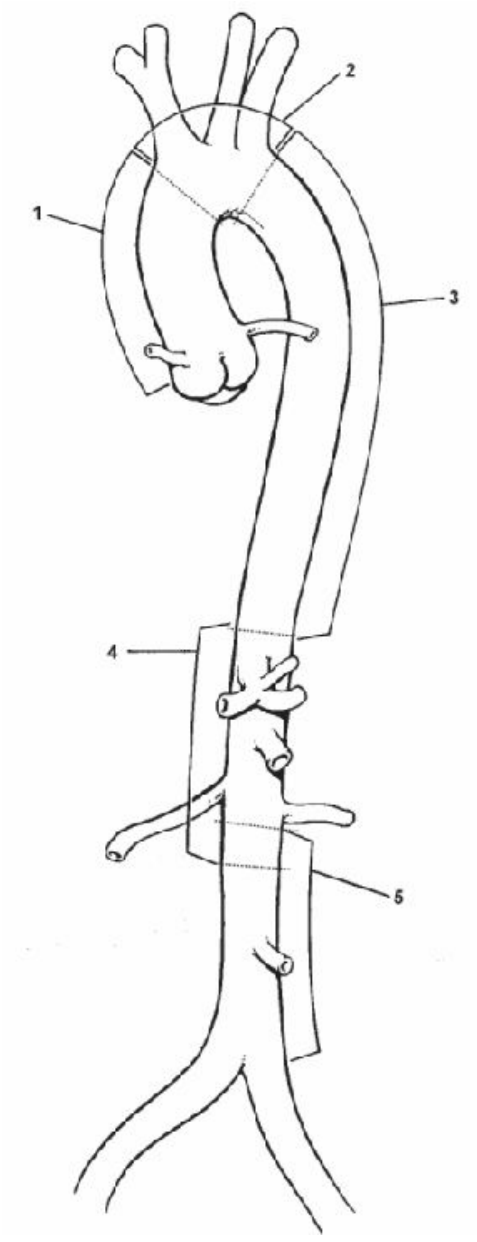

Legenda: 1-Representa a aorta ascendente; 2Representa 0 arco aórtico; 3-Representa a aorta descendente; 4 e 5 Representam a aorta abdominal.

Fonte: SOUZA; ELIAS, (2006).

\section{CLASSIFICAÇÃO DAS DISSECÇÕES AÓRTICAS}

As dissecções aórticas, quando consideradas em relação ao tempo decorrido a partir do momento da dissecção, costumam ser divididas em agudas e crônicas. Consideram-se agudas as dissecções ocorridas há menos de duas semanas; a 
partir desse limite relativamente arbitrário, as dissecções são ditas crônicas (DIAS et al., 2009). A utilidade dessa classificação resulta da extrema gravidade que acompanha as dissecções aórticas. De Bakey e colaboradores em 1965, classificaram as dissecções aórticas em três tipos principais, conforme a localização e a extensão (SOUZA; ELIAS, 2006; DIAS et al., 2015).

- O tipo I corresponde a dissecção que se inicia na aorta ascendente e progride até a aorta descendente ou até o interior do abdome;

- O tipo II corresponde as dissecções que se iniciam na aorta ascendente e se mantém limitadas àquela região

- O tipo III corresponde as dissecções da aorta descendente, que podem ou não se estender à porção subdiafragmática da aorta. No subtipo IIIA a dissecção é restrita à aorta descendente e no subtipo IIIB a dissecção se estende até a aorta abdominal, podendo alcançar ou ultrapassar a sua bifurcação.

Em 1970, um grupo da Universidade de Stanford, introduziu uma classificação mais simples para as dissecções aórticas. Dividindo em dois tipos: A e B. O tipo A corresponde as dissecções em que há envolvimento da aorta ascendente; o tipo B corresponde as dissecções em que há envolvimento da aorta descendente. A classificação de Stanford não se baseia na localização da laceração da íntima e sim no comprometimento das regiões da aorta no processo da dissecção (PAN et al., 2018). O tipo A ou dissecção anterior, compreende as dissecções que têm início na aorta ascendente, enquanto o tipo B ou dissecção posterior compreende as dissecções que se iniciam na aorta descendente, distalmente à origem da artéria subclávia esquerda (BUFFOLO et al., 2018; KIM et al., 2018).

FIGURA 2. Classificações para dissecção da aorta

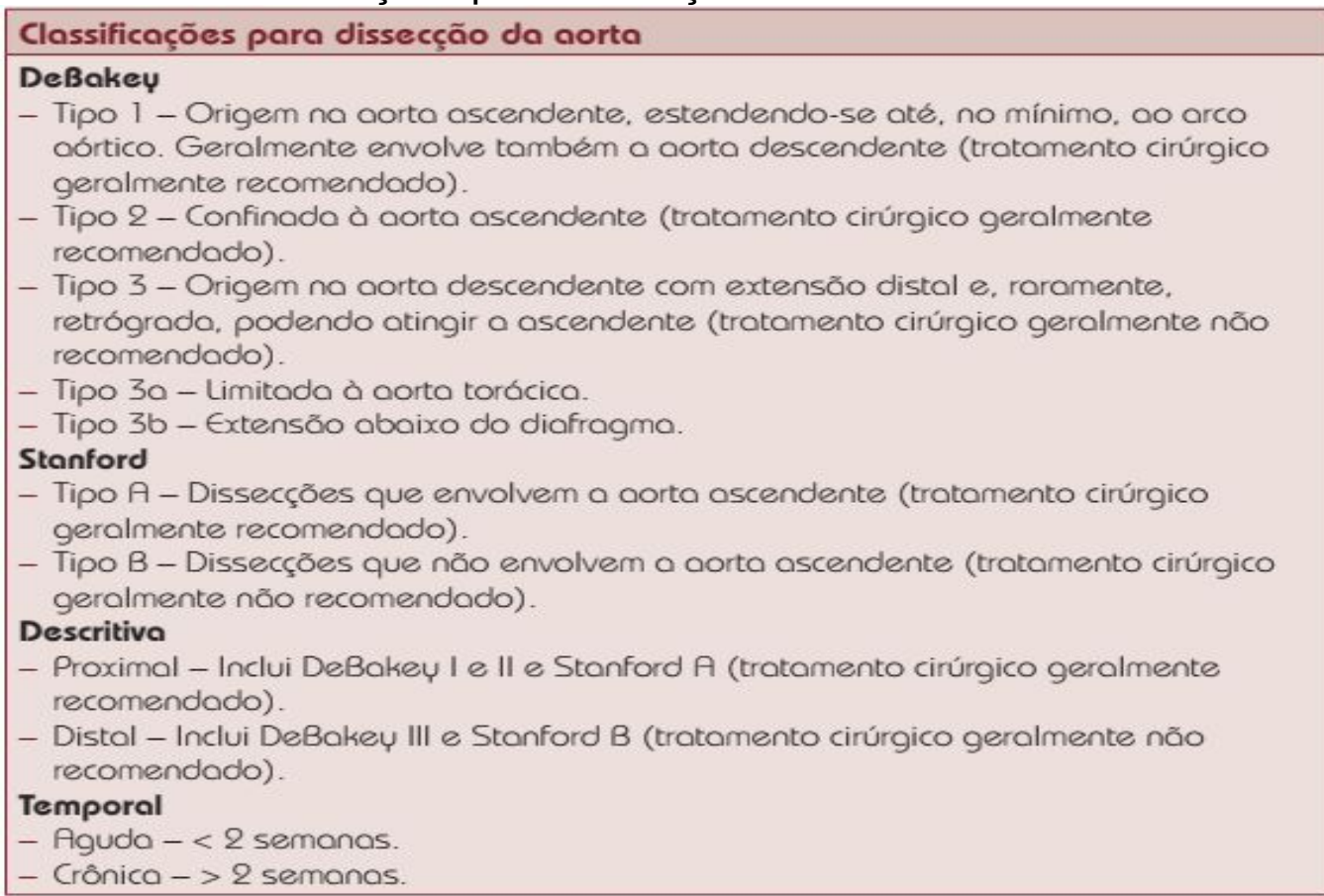

Fonte: KIM et al., (2018). 


\section{INCIDÊNCIA E MORTALIDADE}

Cerca de $50 \%$ a $60 \%$ dos aneurismas da aorta torácica comprometem a aorta ascendente, $30 \%$ a $40 \%$ a aorta descendente, $10 \%$ o arco aórtico e $10 \%$ a porção toracoabdominal. Sem tratamento apropriado, cerca de $50 \%$ dos pacientes com dissecção aórtica falecem nas primeiras 24 horas após o início da dissecção; 25\% sobrevivem a primeira semana e, apenas $10 \%$ dos pacientes sobrevivem seis meses (SOUZA; ELIAS, 2006; NOVAES et al., 2013; ZIMMERMAN et al, 2018). Nos Estados Unidos ocorrem em torno de seis a 10 mil casos por ano. A incidência real é desconhecida, pois muitos pacientes morrem antes da admissão hospitalar ou, ao chegarem, não recebem o diagnóstico correto. Sem tratamento, a mortalidade é elevada: $25 \%$ em 24 horas, $50 \%$ em uma semana e $75 \%$ em 30 dias. Após um ano, 90\% dos pacientes vão a óbito caso não sejam tratados (LEl et al., 2019).

Em um estudo, realizado utilizando a base de dados colhida de uma unidade de terapia intensiva com 30 leitos, pertencente a um hospital de 230 leitos, 11 dos 12 pacientes do grupo com dissecção aguda de aorta tiveram pelo menos uma complicação pós-operatória. Sangramento pós-operatório $50 \%$ e pneumonia hospitalar 40\% (ALMEIDA et al., 2011).

Dissecção aguda da aorta tipo A está associada com uma taxa de mortalidade muito alta entre $1 \%$ e $2 \%$ por hora após o início dos sintomas, se não for tratada, e até $20 \%$ dos pacientes morrem antes de receber atenção médica. $O$ padrão atual de cuidado no tratamento é a cirurgia de emergência, que está associada a uma chance de aproximadamente $70 \%$ de sobrevivência e alta mortalidade e morbidade pós-operatória (HAN et al., 2013).

\section{FATORES DE RISCO PARA MORTALIDADE}

Em um estudo envolvendo um delineamento transversal, com coleta de dados de 257 pacientes pode se observar os principais fatores de risco pré, intra e pósoperatórios para mortalidade hospitalar em pacientes submetidos à cirurgia de aorta (GAWINECKA et al., 2017). Sendo: Etnia negra, doença cerebrovascular, hemopericárdio, revascularização miocárdica cirúrgica associada, revisão de hemostasia e tempo de CEC associaram-se independentemente com risco aumentado de óbito hospitalar (CHRISTI et al., 2017).

A presença de dor torácica associou-se independentemente com risco reduzido de óbito hospitalar. Assim como o uso de antifibrinolítico, complicação renal, pulmonar e tempo de Circulação Extracorpórea prolongado associaram-se independentemente com risco aumentado de desfecho clínico composto (óbito, sangramento, disfunção ventricular ou complicações neurológicas) (DIAS et al., 2009; ISSA et al., 2013). 
FIGURA 3. Fatores de risco para dissecção de aorta

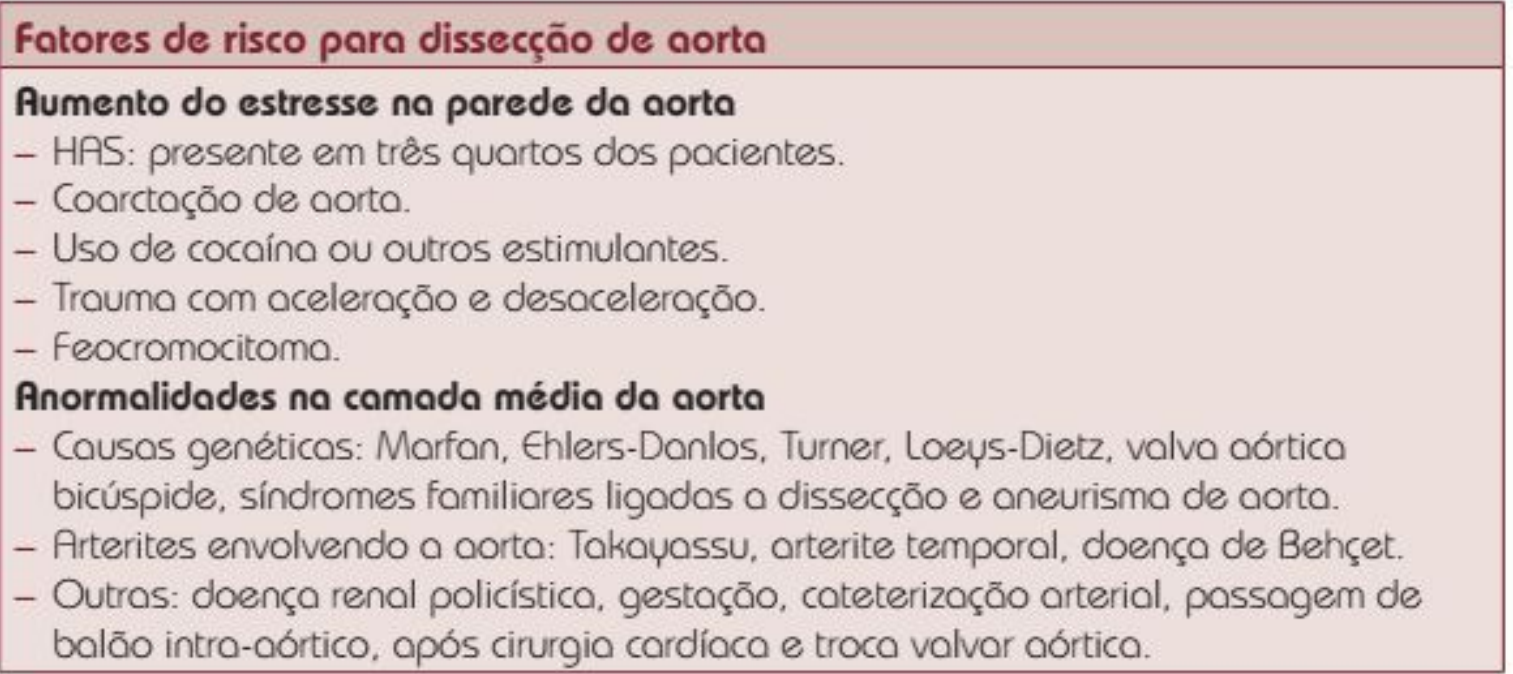

Fonte: SANTOS et al., 2020.

\section{PERFUSÃO PARA CIRURGIAS DA AORTA ASCENDENTE}

As técnicas e táticas de circulação extracorpórea utilizadas para a cirurgia dos aneurismas e das dissecções da aorta ascendente podem, em determinadas circunstâncias, apresentar características especiais que dependem das dimensões e outros aspectos da lesão ou, simplesmente dependem de preferências da equipe cirúrgica e devem ser realizadas o mais precocemente possível (ALMEIDA et al., 2011; PONTES et al., 2013).

A via de acesso aos aneurismas da aorta ascendente é a esternotomia mediana convencional, habitualmente utilizada para a maioria dos procedimentos em cirurgia cardíaca. A esternotomia mediana permite o acesso à aorta, desde a sua origem no ventrículo esquerdo até o início da aorta descendente (ZEN et al., 2020). Em casos extremos, pode ocorrer até a corrosão óssea do esterno e das costelas. Situação semelhante pode existir quando o aneurisma se desenvolve em pacientes anteriormente submetidos à cirurgia cardíaca (DIAS et al., 2015; DEMARTINO et al., 2018). Nesses casos, em geral, o aneurisma não tem a proteção do revestimento pericárdico e pode estar aderido à superfície posterior do esterno. A investigação pré-operatória pela tomografia computadorizada permite demonstrar as relações entre a parede anterior do aneurisma e o esterno e pode ser decisiva no planejamento tático da cirurgia e da perfusão. Quando há risco real ou potencial de rotura do aneurisma durante a incisão do esterno, pode ser necessária a canulação dos vasos femorais, artéria e veia, para a instituição de um bypass temporário que permita a abertura do tórax com menores riscos (SALVIANO et al., 2016)

$\mathrm{Na}$ cirurgia dos aneurismas, mais que nos demais procedimentos, é fundamental que o perfusionista complete a montagem e o enchimento do circuito antes do início da abertura do tórax, devendo permanecer alerta e pronto para a canulação e início da perfusão a qualquer instante, no caso de ocorrer rotura acidental da lesão (FARAG et al., 2019). Embora uma variedade de lesões possa comprometer a aorta ascendente, os aneurismas e as dissecções são as lesões mais frequentemente encontradas e as técnicas operatórias e de circulação extracorpórea são essencialmente as mesmas (SOUZA; ELIAS, 2006; ERBEN et al., 2019). 


\section{SITIOS DE CANULAÇÃO PARA CIRURGIAS DA AORTA ASCENDENTE}

A técnica de canulação varia de acordo com a localização e a extensão da patologia, especialmente em relação a canulação arterial. Uma combinação de canulação da veia femoral e da veia cava superior pode ser usada nos pacientes com instabilidade hemodinâmica que necessitam de suporte circulatório antes da esternotomia e nos pacientes em risco de sofrer lesão aórtica durante, em virtude das grandes dimensões do aneurisma ou do falso lúmen (SOUZA; ELIAS, 2006; OHNO; MINATOYA, 2020).

Em certos casos, especialmente nas cirurgias de emergência pode ser utilizada a canulação extratorácica, em que a veia cava inferior é canulada através da veia femoral e a veia cava superior é canulada através da veia jugular interna (KIM et al., 2018; CANNING et al., 2019). Entretanto, nos pequenos aneurismas restritos à raiz e à porção média da aorta, a parede aórtica próxima ao ponto onde emerge o tronco braquiocefálico pode ser usada para inserção da cânula arterial, evitando a incisão e dissecção adicionais da região femoral (FONTES et al., 2005; SOUZA; ELIAS, 2006).

Independente da cirurgia programada, a canulação para a descompressão das cavidades esquerdas e aspiração do sangue intra-cardíaco, depende das preferências da equipe, mas é de grande importância nos casos em que há insuficiência aórtica, para evitar a rápida dilatação do ventrículo esquerdo no início da perfusão, reduzir o reaquecimento do miocárdio, facilitar a exposição da válvula aórtica e auxiliar a retirada do ar intra-cardíaco ao final da operação(SOUZA; ELIAS, 2006; SALVIANO et al., 2016).

\section{PERFUSÃO PARA CIRURGIAS DA AORTA DESCENDENTE}

O tratamento convencional das dissecções da aorta descendente, tipo $\mathrm{B}$ de Stanford, inclui tanto o tratamento clínico quanto a abordagem cirúrgica. O tratamento inicial para as dissecções agudas não complicadas é medicamentoso, enquanto que, quando associada às complicações de dor persistente, ruptura tamponada, isquemia de membros ou órgãos, o tratamento é cirúrgico (DIAS et al., 2007; NOVERO et al., 2012).

O tratamento cirúrgico e o suporte circulatório das lesões da aorta descendente dependem da extensão da lesão (ROBISON et al., 2017; GUPTA et al., 2018). As lesões segmentares confinadas ao interior da cavidade torácica são abordadas através de uma toracotomia posterolateral esquerda, enquanto as lesões que comprometem a aorta tóraco-abdominal combinam a toracotomia e 0 acesso abdominal através de laparotomia e abordagem extraperitonial (SOUZA; ELIAS, 2006; QUINTAS et al., 2016; BADGER et al., 2017). Também preferível em casos de re-operações, por ser uma opção válida quando o coração está muito aderido ao esterno.

A entrada em CEC previamente a esternotomia ajuda a evitar lesões cardíacas graves. A artéria femoral pode ser canulada via punção ou diretamente por dissecção (CHISHTI et al., 2017; GUPTA et al., 2018). Vale notar que para pacientes com aneurisma, dissecções ou trombos murais na aorta descendente, a manutenção da CEC via artéria femoral acarreta em maior risco de AVC devido à chance de embolia / dissecção pelo fluxo retrógrado (KIM et al., 2018). Outros sítios são possíveis como o próprio arco aórtico (guiado por ECO transesofágico e via Seldinger) e a artéria braquial (DIAS et al., 2007). 
A canulação pode ser feita diretamente na artéria ou anastomosando um tubo de dacron de 8 ou $10 \mathrm{~mm}$ término-lateral (NOVERO et al., 2012). A anastomose do tubo de dacron permite manter adequada perfusão distal do sítio canulado. Ainda, a canulação direta está associada a maior taxas de eventos adversos como dissecção da artéria, fluxo distal insuficiente, embolias, dissecção proximal ao local de inserção (pela ponta da cânula próxima a parede de uma artéria fragilizada). Com a escolha do sítio de canulação arterial ideal, a cirurgia de troca de aorta ascendente / arco aórtico pode acontecer tranquilamente, com a segurança de um bom fluxo arterial e mínimo risco de acidentes (SOUZA; ELIAS, 2006; QUINTAS et al., 2016; BADGER et al., 2017).

\section{SITIOS DE CANULAÇÃO PARA CIRURGIAS DA AORTA DESCENDENTE}

Apesar das controvérsias em relação ao tratamento ideal na abordagem inicial das dissecções tipo B de Stanford, o tratamento clínico dos pacientes não complicados ainda prevalece e dentre as várias estratégias cirúrgicas em uso para a proteção medular destacam-se a cirurgia mediante o simples clampeamento aórtico acima e abaixo da lesão, com ou sem a aplicação de um shunt intra-aórtico (shunt de Gott), o emprego do " bypass " esquerdo, a perfusão veno-arterial parcial (distal), mediante o clampeamento dos vasos femorais e a parada circulatória hipotémica (SOUZA; ELIAS, 2006; DIAS et al., 2007; CHEN et al., 2019).

O bypass esquerdo constitui uma forma de suporte circulatório capaz de facilitar o tratamento cirúrgico das lesões da aorta descendente (CHEN et al., 2019). $\mathrm{Na}$ sua forma mais comum, o sangue oxigenado é retirado do átrio esquerdo e, mediante uma bomba propulsora (roletes ou centrífuga), injetado na artéria femoral. A canulação é simples e consiste de duas cânulas, uma para o átrio esquerdo $e$ outra para a artéria femoral, além da bomba propulsora (SOUZA; ELIAS, 2006; DIAS et al., 2007).

A perfusão veno-arterial distal, também conhecida como perfusão femorofemoral, consiste em remover o sangue venoso pela veia femoral até um oxigenador e na infusão do sangue oxigenado pela artéria femoral (DIAS et al., 2007; HEALEY et al., 2017).

\section{TRATAMENTO}

De forma geral, o envolvimento da aorta ascendente é indicativo de tratamento cirúrgico. Quando apenas a aorta descendente é envolvida, inicialmente o tratamento será clínico (BADERKHAN et al., 2016). O objetivo principal da cirurgia no tratamento de dissecção aguda da aorta ascendente é para prevenir a ruptura da dissecção e subsequente hemorragia. Para pacientes com envolvimento da raiz aórtica, existem dois métodos convencionais de tratamento cirúrgico (HAN et al., 2013; SANTOS et al., 2020).

Primeiro, se na raiz aórtica tem evidência de patologias da válvula aórtica, do anel aórtico, ou há um aneurisma aórtico existente, uma válvula sparing ou a abordagem Bentall podem ser usados. Segundo, se as patologias acima mencionadas não forem aparentes, a substituição da aórtica ascendente com reconstrução tradicional da raiz da aorta (substituição supracomissural) pode ser realizada (REIMERINK et al., 2003; BIANCARI et al., 2011). Várias modificações de abordagens de preservação da válvula aórtica também foram descritas e realizadas, incluindo remodelação, remodelação com Teflon, colagem de camadas dissecadas e 
substituição da aorta ascendente, supracoronária com reconstrução da raiz. A preservação da válvula aórtica pode reduzir a curto e longo prazo, complicações associadas a substituições de válvulas mecânicas e biológicas (HAN et al., 2013; TAN et al., 2017).

Aneurismas pequenos crescem a uma taxa variável: Embora a expansão média seja estimada em 0,4 cm/ano, a evolução de um indivíduo específico não pode ser prevista devido à grande variabilidade (NEILSON et al., 2018). A cirurgia eletiva leva ao risco de morte ou complicações maiores, que dependem da experiência do cirurgião do grupo e da quantidade de cirurgias no hospital, mas, nos últimos tempos, a taxa de mortalidade deve ser inferior a 5\%. Embora análises de pesquisas recentes indiquem que a taxa média de mortalidade é de 3,5\%, diferenças de até $10 \%$ podem ser observadas entre cirurgiões ou instituições (SWEETING et al., 2018).

A presença de sintomas é uma indicação cirúrgica voluntária, independentemente do diâmetro, e inclui dor/desconforto na cintura ou abdominal, embolia distal ou compressão das estruturas vizinhas (AZIZ et al., 2016; CANNING et al., 2019). Obviamente, a cirurgia de emergência é necessária para pacientes com suspeita de ruptura; para aneurismas inflamatórios, independentemente do tamanho, eles devem ser corrigidos seletivamente porque são acompanhados por manifestações sistêmicas óbvias, como febre e perda de peso; para aneurismas assintomáticos, os pacientes devem tomar medidas de intervenção a considerar a relação entre o risco de ruptura e o risco da cirurgia individual e a expectativa de vida (REIMERINK et al., 2013). Na década de 1990, era a recomendação da Sociedade Internacional de Cirurgia Vascular para cirurgia eletiva. A letalidade dos pacientes com DPOC é de $5,0 \mathrm{~cm}$ ou mesmo $4,0 \mathrm{~cm}$, que se baseia na baixa mortalidade pós-operatória (1\%) desse grupo (SAN NOBERTO et al., 2016).

Também é claramente recomendado não contraindicar a cirurgia de qualquer diâmetro em casos de tumores metastáticos, insuficiência cardíaca grave ou outra doença que limite a sobrevida por não mais do que dois a três anos. No entanto, em dois estudos recentes de resultados clínicos importantes, eles podem precisar revisar esses padrões (FUJIMURA et al., 2018).

\section{CONCLUSÃO}

Não existe um consenso sobre a canulação arterial mais indicada para cada caso específico quando se trata de dissecção de aorta, principalmente do tipo B. Elementos importantes para estratégias de canulação são o estabelecimento rápido de fluxo sistêmico suficiente, cuidados com a má perfusão existente no préoperatório e prevenção de nova má perfusão induzida pela CEC. As complicações neurológicas não devem ser ignoradas e a mudança para uma canulação diferente pode ser necessária se houver algum sinal de má perfusão. O requisito mais importante é o monitoramento constante durante a cirurgia e na CEC. Levando esses aspectos em consideração, assim como a escassez de artigos, livros e outras publicações referentes à Circulação Extracorpórea nos casos de Dissecção de aorta (tanto ascendente quanto descente), bem como dos sítios de canulação e estratégias de tratamento cirúrgico, tradicionais e alternativos. Observa-se a necessidade de maiores estudos. 


\section{REFERÊNCIAS}

ALMEIDA, G. F.; JAPIASSU, R.; KURTZ, A. M.; DRUMOND, P.; FREITAS, L. E. et al. Complicações pós-operatórias de pacientes com dissecção de aorta ascendente tratados cirurgicamente. Revista Brasileira de Terapia Intensiva, v. 23, n. 3, p. 304-311, 2011. Disponível em: https://doi.org/10.1590/s0103-507x2011000300008

AZIZ F.; ILLIG, A.; CALERO, A.; SMEDS, R. M.; CHARUTON-OW, M. et al. Ruptured abdominal aortic aneurysm: Is endovas-cular aneurysm repair the answer for everybody? Seminarys in Vascular Surgery, v. 29, n. 4, p. 35-40, 2016. Disponível em: DOI: 10.1053/j.semvascsurg.2016.04.002

BADGER, S.; FORSTER, R.; BLAIR, H. P.; ELLIS, P.; KEE, F. et al. Endovascular treatment for ruptured abdominal aortic aneurysm (Review). Co-chrane Data Base Systemic Review, v. 5, n. 2, p. 45-62, 2017. Disponível em: DOI: 10.1002/14651858.CD005261.pub4

BADERKHAN, H.; GONÇALVES, M. B. F.; OLIVEIRA, N. G.; VERHAGEN, H. J. M.; WANHAINEN, A. et al. Challeng-ing anatomy predicts mortality and complications after endovascular treatment of ruptured abdominal aortic aneurysm. Journal of Endovascular Therapy, v. 23, n.4, p. 919-927, 2016. Disponível em: DOl: 10.1002/14644678.CD005261.pub5

BIANCARI, F.; VASQUES, F.; BENENATI, V.; JUVOVEN, T. Contemporary results after surgical repair of type $A$ aortic dissection in patients aged 80 years and older: a systematic review and meta-analysis. Europe Journal of Cardiothoracic Surgery, v. 40, n. 5, p. 1058-1063, 2011. Disponível em: doi: 10.1016/j.ejcts.2011.03.044.

BUFFOLO, E.; DE ALMEIDA, J. H.; FONSECA, P. Evolução No Tratamento Dos Aneurismas Da Aorta Torácica. Revista da Sociedade Brasileira de Cardiologia de São Paulo, v. 28, n. 1, p. 66-70, 2018.

CANNING, P. TAWFICK, W.; KAMEL, K.; HYNES, N.; SULTAN, S. et al. Q-TWiST and cost-ef-fectiveness analysis of endovascular versus open repair for ruptured abdominal aortic aneurysms in a high deliberate practice volume center. Annals of Vascular Surgery, v. 56, n. 2, p. 163-174, 2019. Disponível em: DOI: 10.1016/j.avsg.2018.08.091

CHEN, S. L.; KABUTEY, N.; WHEALON, M. D.; KUO, I. J.; DONAYRE, C. E. et al. Locoregional anesthesia offers improved outcomes after endovascular repair of ruptures abdominal aortic aneurysms. Annals of Vascular Surgery, v. 59, n. 2, p. 134-142, 2019. Disponível em: $\square$ DOI: 10.1016/j.avsg.2018.12.083

CHISHTI, M. A.; RAWAT, V.; NAGARWAL, P. Acute Dissection of the Ascending Aorta: A Case Report and Topic Review. Journal of Mahatma Gandhi University of Medical Sciences and Technology, v. 2, n. 1, p. 31-34, 2017. Disponível em: DOI: 10.5005/jp-journals-10057-0028

COSTA, J. M.; PAPA, F. de V.; STASZKO, K. F. Aortic dissection after mitral valve 
replacement: the role of intraoperative echocardiography in the diagnosis. Brazilian Journal of Anesthesiology, v. 69, n. 2, p. 197-199, 2019. Disponível em: https://doi.org/10.1016/j.bjan.2018.05.001

DEMARTINO, R. R.; SEN, I.; HUANG, Y.; BOWER, T. C.; ODERICH, G. S et al. Population-Based Assessment of the Incidence of Aortic Dissection, Intramural Hematoma, and Penetrating Ulcer, and Its Associated Mortality from 1995 to 2015. Circulation and Cardiovascular Quality and Outcomes, v. 11, n. 8, 2018. Disponível em: DOI: 10.1161/CIRCOUTCOMES.118.004689

DIAS, R. R.; JUDAS, G.; OLIVEIRA, M. A. P.; MALBOUISSON, L. M. S.; FIORELLI, A. I. et al. Há espaço para o tratamento endovascular nas dissecções crônicas da aorta descendente? Revista Brasileira de Cirurgia Cardiovascular, [s. I.], v. 22, n. 4, p. 441-447, 2007. Available at: https://doi.org/10.1590/s010276382007000400010

DIAS, R. R.; DUNCAN, J. A.; VIANNA, D. S.; FARIA, L. B.; RAMIREZ, F. F. A. et al Surgical treatment of complex aneurysms and thoracic aortic dissections with the Frozen Elephant Trunk technique. Revista Brasileira de Cirurgia Cardiovascular, v. $30, \quad$ n. 2, p. 205-210, 2015. Disponível em: http://www.scielo.br/scielo.php?script=sci_arttext\&pid=S01026382015000200011\&Ing=en. doi: 10.5935/1678-9741.20140119.

DIAS, R. R.; STOLF, N. A. G. Doenças da Aorta Torácica. Clínica médica: doenças cardiovasculares, doenças respiratórias, emergências e terapia intensiva, $p$. 360-367, 2009.

ERBEN, Y.; ODERICH, G. S.; VERHAGEN, H. J. M.; WITSENBURG, M.; HOVEN, A. T. V. D. et al. Multicenter experience with endovascular treatment of aortic coarctation in adults. Journal of Vascular Surgery, v. 69, n. 3, p. 671-679, 2019. Disponível em: DOI: 10.1016/j.jvs.2018.06.209

FARAG, E. S.; KLUIN, J.; HEER, F. D.; AHMED, Y.; SOJAK, V.; et al. Aortic coarctation repair through left thoracotomy: results in the modern era. Europe Journal of Cardiothoracic Surgery, v. 55, n. 2, p. 331-337, 2019. Disponível em: https://doi.org/10.1093/ejcts/ezy241

FONTES, R. D.; DIAS, C. A.; OSHIRO, M.; CARNEIRO, G. L.; VELLOSO, C. et al. Perfusão cerebral retrógrada e anterógrada no tratamento cirúrgico de dissecção aguda da aorta ascendente. Revista Brasileira de Cirurgia Cardiovascular, v. 20, n. 2, p. 186-188, 2005. Disponível em: https://doi.org/10.1590/s010276382005000200015

FOX, N.; SCHWARTZ, D.; SALAZAR, J. H.; HAUT, E. R.; DAHM, P. et al. Evaluation and management of blunt traumatic aortic injury: a practice management guideline from the Eastern Association for the Surgery of Trauma. Journal of Trauma Acute and Care Surgery, v. 78, n. 1, p. 136-146, 2015. Disponível em: http://dx.doi.org/10.1097/TA.0000000000000470. PMid:25539215 
FUJIMURA, N.; TAKAHARA, M.; ISOGAI, N.; YASHIRO, H.; SHIBUTANI, S. Retroperitone-al hematoma volume is a good predictor of periop-erative mortality after endovascular aneurysm repair for ruptured abomdinal aortic aneurysm. Journal of Vascular Surgery, v. 68, n. 2, p. 998-1006, 2018. Disponível em: DOI: 10.1016/j.jvs.2018.01.048

GARCíA, R. M. E.; MARTINS, G. G.; VALENZUELA, V. F.; GONZALEZ, M. D.; LEBRUN, J. M. Long-term outcomes of thoracic endovascular aortic repair focused on bird beak and oversizing in blunt traumatic thoracic aortic injury. Annals of Vascular Surgery, v. 50, n. 4, p. 140-147, 2018. Disponível em: http://dx.doi.org/10.1016/j.avsg.2018.02.001. PMid:29455010

GAWINECKA, J.; SCHONRATH, F.; ECKARDSTEIN, A. Acute aortic dissection: pathogenesis, risk factors, and diagnosis. Swiss Medicine, v. 2, n. 1, p. 147-158, 2017. Disponível em: DOI: 10.4414/smw.2017.14489

GUPTA, A. L.; ARIDI, H. D.; LOCHAM, S.; NEJIM, B.; VEITH, F. J. et al. Real-world evidence of superiority of endovascular repair in treat-ing ruptured abdominal aortic aneurysm. Journal of Vascular Surgery, v. 68, n. 2, p. 74-81, 2018. Disponível em: DOI: $10.1016 / j . j v s .2017 .11 .065$

HAN, Q. Q.; SONG, Z.; LU, F. L.; LANG, Z.; XU, L. M. et al. Reconstrução da raiz da aorta reforçada para dissecção aguda da aorta tipo a envolvendo a raiz da aórtica. Brazilian Journal of Cardiovascular Surgery, v. 28, n. 2, p. 190-199, 2013. Disponível em: https://doi.org/10.5935/1678-9741.20130028

HEALEY, C. T.; NEILSON, M.; CLARK, D.; SCHANZER, A.; ROBINSON, W. Predicting mortality of ruptured abdominal aortic aneurysms in the era of endovascular repair. Annals of Vascular Surgery, v. 38, n. 2, p. 59-63, 2017..Disponível em: DOI: 10.1016/j.avsg.2016.09.006

ISSA, M.; AVEZUM, A.; CHAGAS, D.; SANCHES, A. F. BENTO, L. C. et al. Fatores de risco pré, intra e pós-operatórios para mortalidade hospitalar em pacientes submetidos à cirurgia de aorta. Brazilian Journal of Cardiovascular Surgery, v. 28, n. 1, p. 10-21, 2013. Disponível em: https://doi.org/10.5935/1678-9741.20130004

KIM, E. S. H.; BECKMAN, J.; LU, X.; ZENG, K.; XU, A. et al. Takayasu arteritis: challenges in diagnosis and management. Heart, v. 104, n. 7, p. 558-565, 2018. Disponível em: DOI: 10.1136/heartjnl-2016-310848

LEI, P.; YAN, J.; GAO, F. U.; HAN, S. S.; CAO, G. J. Case report of a 28-year-old man with aortic dissection and pulmonary shadow due to granulomatosis with polyangiitis. BMC Pulmonary Medicine, v. 19, n. 122, p. 51-62, 2019. Disponível em: https://www.ncbi.nlm.nih.gov/pmc/articles/PMC6615146/

MARCACCIO, C. L.; DUMAS, R. P.; HUANG, Y.; YANG, W.; HOLENA, D. Delayed endovascular aortic repair is associated with reduced in-hospital mortality in patients with blunt thoracic aortic injury. Journal of Vascular Surgery, v. 68, n. 1, p.64-73, 2018. Disponível em: DOI: 10.1016/j.jvs.2017.10.084 
MCCLURE, R. S.; BROGLY, S. B.; LAJKOZ, K.; PAYNE, D.; HALL, S. F. et al. Epidemiology and management of thoracic aortic dissections and thoracic aortic aneurysms in Ontario, Canada: a population-based study. The Journal of Thoracic and Cardiovascular Surgery, v. 155, n. 6, p. 2254-2264, 2018. Disponível em: DOI: 10.1016/j.jtcvs.2017.11.105

MELVINSDOTTIR, I. H.; LUND, S. H.; AGNARSSON, B. A.; SIGVALDASON, K.; GUDBJARTSSON, T. The incidence and mortality of acute thoracic aortic dissection: results from a whole nation study. Europe Journal of Cardiothoracic Surgery, v. 50, n. 6, p. 1111-1117, 2018. Disponível em: DOI: 10.1093/ejcts/ezw235

NEILSON, M.; HEALY, C.; CLARK, D.; NOLAN, B.; JOSH, B. et al. External validation of a rapid ruptured abdominal aortic aneurysm score. Annals of Vascular Surgery, v. 46, n. 2, p. 162-167, 2018. Disponível em: DOI: 10.1016/j.avsg.2017.08.016

NOVAES, F. R.; NAVARRO, T. P.; BERNARDES, R. C.; ROQUETE, F. A.; LIMA, L. C. M. et al. Resultados do anel intraluminal de Castro Bernardes nas cirurgias de aneurismas e dissecções da aorta ascendente. Brazilian Journal of Cardiovascular Surgery, v. 28, n. 2, p. 176-182, 2013. Disponível em: https://doi.org/10.5935/1678-9741.20130026

NOVERO, E. R.; METZGER, P. B.; OBREGON, J.; MARCO, V. L. A.; ROSSI, F. H. et al. Tratamento endovascular das doenças da aorta torácica: Análise dos resultados de um centro. Radiologia Brasileira, v. 45, n. 5, p. 251-258, 2012. Disponível em: https://doi.org/10.1590/S0100-39842012000500004

OHNO, N.; MINATOYA, K. Arterial cannulation to establish cardiopulmonary bypass during surgery for acute aortic dissection. Surgery Today, v. 50, n. 11, p. 13531359, 2020. Disponível em: https://doi.org/10.1007/s00595-019-01921-7

PAN, E.; KYTO, V.; SAVUNEN, T.; GUNN, J. OHNO, N. et al. Early and late outcomes after open ascending aortic surgery: 47-year experience in a single centre. Heart and vessels, v. 33 , n. 4, p. 427-433, 2018. Disponível em: DOI: $10.1007 / \mathrm{s} 00380-017-1075-3$

PLATZZ, J. J.; FABRICANT, L.; NOROTSKY, M.; GUNN, J. KYTO, V. et al. Thoracic trauma: injuries, evaluation, and treatment. Surgery Clinical North American, v. 97, n. 4, p. 783-799, 2017. Disponível em: http://dx.doi.org/10.1016/j.suc.2017.03.004. PMid:28728716

PONTES, J. C. D. V.; MONTSERRAT, A.; DIAS, A. S.; DUARTE, J. J.; BENFATTI, R. A. et al. Correção endovascular de dissecção de aorta ascendente. Brazilian Journal of Cardiovascular Surgery, v. 28, n. 1, p. 145-147, 2013. Disponível em: https://doi.org/10.5935/1678-9741.20130018 
QUINTAS, A.; GONÇALVES, F. B.; RODRIGUES, H.; FERREIRA, R.; RODRIGUES, G. et al. Tratamento endovascular de patologia da aorta torácica: experiência institucional. Angiologia e Cirurgia Vascular, v. 12, n. 1, p. 3-11, 2016. Disponível em: https://doi.org/10.1016/j.ancv.2015.12.002

RAIVIO, P.; SUOJARANTA-YLINEN, R.; KUITUNEN, A. H. Recombinant factor VIla in the treatment of postoperative hemorrhage after cardiac surgery. Annals of Thoracic Surgery, v. 2, n. 5, p. 66-71, 2005. Disponível em: https://www.annalsthoracicsurgery.org/article/S0003-4975(05)00312-7/pdf

REIMERINK, J. J.; VAN DER LAAN, M. J. KOELEMAY, M. J.; BALM, R.; LEGEMATE, D. A. et al. Sys-tematic review and meta-analysis of population-based mortality from ruptured abdominal aortic aneurysm. Brazilian Journal of Surgery, v. 12, n. 3, p. 100-121, 2013. Disponível em: DOI: 10.1002/bjs.9235

ROBINSON, W. P.; OLIVEIRA-PINOT, J.; SOUSA, J.; MANSILHA, A.; PERTESSON, B. et al. Open versus endovascular repair of rup-tured abomdinal aortic aneurysms: What have we learned after more than 2 decades of ruptured aneurysm repair? Surgery, v. 162 , n. 2, p. 1207-1218, 2017. Disponível em: DOI: 10.1016/j.surg.2017.08.008

SALVIANO, A.; EROLES, G. M. G.; SILVA, F. M.; PINTO E SILVA, A. M. R.; RIVETTI, L. et al. Via de acesso alternativa para circulação extracorpórea na dissecção aguda de aorta ascendente aorta. Arquivos médicos, v. 2, n. 1, p. 3540, 2016.

SANTOS, E. C. L.; MASTROCOLA, F.; FIGUINHA, F. C. R.; LIMA, A. G.; BASTOS, E. et al. Manual de Cardiologia. $2^{\circ}$ Edição, Editora Atheneu, 2020.

SAN-NORBERTO, E. M.; FUENTE, R.; GARCIA, I.; REVILLA, A.; PEDROSA, M.; VAQUERO, $C$. et al. New scale for predicting mortality in ruptured abdominal aortic an-eurysms. Circulation and Especialist surgery, v. 4, n. 94, p. 339-345, 2016. Disponível em: DOI: 10.1016/j.cireng.2016.07.005

SEDRAKYAN, A.; WU, A.; SEDRAKYAN, G.; WEST, M. D.; TRANQUILI, M. et al. Aprotinin use in thoracic aortic surgery: safety and outcomes.

Journal of Thoracic and Cardiovasculuar Surgery. v. 2, n. 132, p. 909-917, 2006. Disponível em: https://www.jtcvs.org/article/S0022-5223(06)01225-6/pdf

SCALEA, T. M.; FELICIANO, D. V.; DUBOSE, J. J.; OTTOCHIAN, M.; CONNOR, J. et al. Blunt thoracic aortic injury: endovascular repair is now the standard. Journal of American College of Surgery, v. 228, n. 4, p. 605-610, . 2019. Disponível em http://dx.doi.org/10.1016/j.jamcollsurg.2018.12.022. PMid:30630086.

SOUZA, M. H.; ELIAS, D. Fundamentos da Circulação Extracorpórea. 2. ed. Rio de Janeiro, RJ - Brasil: Centro Editorial Alfa Rio, 2006.

SWEETING, M. J.; ULUG, P.; ROY, J.; HULTGREN, R.; BALM, R. et al. Value of risk 
scores in the decision to palliate patients with ruptured abdom-inal aortic aneurysm. The British Journal of Surgery, v. 105, n. 2, p. 1135-1144, 2018. Disponível em: DOI: $10.1002 /$ bjs. 10820

TAN, T. W.; ESLAMI, M.; RYBIN, D.; DOROS, G.; ZHANG, W. W. et al. Outcomes of endovascu-lar and open surgical repair of ruptured abdominal aortic aneurysms in elderly patients. Journal of Vascular Surgery, v. 66, n. 5, p. 64-70, 2017. Disponível em: DOI: 10.1016/j.jvs.2016.10.119

ZIMMERMAN, K. P.; ODERICH, G.; POCHETTINO, A.; HANSON, K. T.; HABERMANN, E. B. et al. Improving mortality trends for hospitalization of aortic dissection in the National Inpatient Sample. Journal of Vascular Surgery, v. 64, n. 3, p. 606-615, 2018. Disponível em: DOI: 10.1016/j.jvs.2016.03.427 